\title{
Network-constrained AC unit commitment under uncertainty: A Benders' decomposition approach
}

\author{
Nasri, Amin; Kazempour, Seyyedjalal; Conejo, Antonio J.; Ghandhari, Mehrdad
}

Published in:

IEEE Transactions on Power Systems

Link to article, DOI:

10.1109/TPWRS.2015.2409198

Publication date:

2015

Document Version

Peer reviewed version

Link back to DTU Orbit

Citation (APA):

Nasri, A., Kazempour, S., Conejo, A. J., \& Ghandhari, M. (2015). Network-constrained AC unit commitment under uncertainty: A Benders' decomposition approach. IEEE Transactions on Power Systems, 31(1), 412 - 422 .https://doi.org/10.1109/TPWRS.2015.2409198

\section{General rights}

Copyright and moral rights for the publications made accessible in the public portal are retained by the authors and/or other copyright owners and it is a condition of accessing publications that users recognise and abide by the legal requirements associated with these rights.

- Users may download and print one copy of any publication from the public portal for the purpose of private study or research.

- You may not further distribute the material or use it for any profit-making activity or commercial gain

- You may freely distribute the URL identifying the publication in the public portal 


\section{Network-Constrained AC Unit Commitment Under Uncertainty: A Benders' Decomposition Approach}

Amin Nasri, Student Member, IEEE, S. Jalal Kazempour, Member, IEEE, Antonio J. Conejo, Fellow, IEEE, and Mehrdad Ghandhari, Senior Member, IEEE

\begin{abstract}
This paper proposes an efficient solution approach based on Benders' decomposition to solve a network-constrained ac unit commitment problem under uncertainty. The wind power production is the only source of uncertainty considered in this paper, which is modeled through a suitable set of scenarios. The proposed model is formulated as a two-stage stochastic programming problem, whose first-stage refers to the day-ahead market, and whose second-stage represents real-time operation. The proposed Benders' approach allows decomposing the original problem, which is mixed-integer nonlinear and generally intractable, into a mixed-integer linear master problem and a set of nonlinear, but continuous subproblems, one per scenario. In addition, to temporally decompose the proposed ac unit commitment problem, a heuristic technique is used to relax the inter-temporal ramping constraints of the generating units. Numerical results from a case study based on the IEEE one-area reliability test system (RTS) demonstrate the usefulness of the proposed approach.
\end{abstract}

Index Terms-Benders' decomposition, network-constrained ac unit commitment, stochastic programming, wind power uncertainty.

\section{NOTATION}

The main notation used is defined below. All variables and constants are expressed in per-unit. Symbols $\mathbf{v}$ and $\boldsymbol{\theta}$ written in bold without index are vector forms of the nodal voltage magnitude and the nodal voltage angles, respectively.

1) Indices:

$d \quad$ Index for loads.

$i \quad$ Index for generating units.

$k \quad$ Index for wind farms.

$n / m \quad$ Indices for system nodes.

Manuscript received July 08, 2014; revised October 07, 2014, December 13, 2014, and February 09, 2015; accepted February 26, 2015. The work of A. Nasri was supported by Erasmus Mundus Joint Doctorate in Sustainable Energy Technologies and Strategies . The work of S. J. Kazempour was supported by the US National Science Foundation under grant ECCS 1230788. Paper no. TPWRS-00930-2014.

A. Nasri and M. Ghandhari are with the KTH Royal Institute of Technology, Stockholm, Sweden (e-mail: Amin.Nasri@ee.kth.se; Mehrdad.Ghandhari@ee. kth.se).

S. J. Kazempour is with the Technical University of Denmark, Kgs. Lyngby, Denmark (e-mail: seykaz@elektro.dtu.dk).

A. J. Conejo is with The Ohio State University, Columbus, OH 43210 USA (e-mail: conejonavarro.1@osu.edu).

Color versions of one or more of the figures in this paper are available online at http://ieeexplore.ieee.org.

Digital Object Identifier 10.1109/TPWRS.2015.2409198 $s \quad$ Index for wind power scenarios.

$t \quad$ Index for time periods.

2) Sets:

$\mathcal{D} \quad$ Set of loads.

$\mathcal{G} \quad$ Set of generating units.

$\mathcal{K} \quad$ Set of wind farms.

$\Omega_{n} \quad$ Set of nodes adjacent to node $n$.

Sets $\mathcal{K}, \mathcal{D}$, and $\mathcal{G}$ include subscript $n$ if referring to the set of wind farms, loads and generating units, respectively, located at node $n$.

3) Continuous Variables:

$C_{i t}^{\mathrm{SU}} \quad$ Start-up cost of unit $i$ in period $t$.

$P_{i t}^{\mathrm{DA}} \quad$ Active power scheduled for unit $i$ in period $t$.

$W_{k t}^{\mathrm{DA}} \quad$ Active power scheduled for wind farm $k$ in period

$\theta_{n t}^{\mathrm{DA}} \quad$ Voltage angle at node $n$ in period $t$ at the scheduling stage.

$L_{d t s}^{\mathrm{SH}} \quad$ Involuntarily active load shedding of load $d$ in

period $t$ and scenario $s$.

$Q_{i t s} \quad$ Reactive power output of unit $i$ in period $t$ and scenario $s$.

$r_{i t s} \quad$ Reserve deployed by unit $i$ in period $t$ and scenario $s$.

$W_{k t s}^{\mathrm{SP}} \quad$ Wind power spillage of wind farm $k$ in period $t$ and scenario $s$.

$v_{n t s} \quad$ Voltage magnitude at node $n$ in period $t$ and scenario $s$.

$\theta_{n t s} \quad$ Voltage angle at node $n$ in period $t$ and scenario $s$.

Note that the first four variables pertain to the first-stage.

4) Binary Variables:

$u_{i t} \quad 0 / 1$ variable that is equal to 1 if unit $i$ is scheduled to be committed in period $t$.

5) Constants:

$\rho_{s} \quad$ Probability of scenario $s$.

$\lambda_{i}^{\mathrm{SU}} \quad$ Start-up cost of unit $i$.

$C_{i} \quad$ Marginal cost of the energy offered by unit $i$.

$L_{d t}^{\mathrm{P}} \quad$ Active power consumed by load $d$ in period $t$.

$L_{d t}^{\mathrm{Q}} \quad$ Reactive power consumed by load $d$ in period $t$.

$\bar{P}_{i} \quad$ Active power capacity of unit $i$. 
This article has been accepted for inclusion in a future issue of this journal. Content is final as presented, with the exception of pagination.

$\underline{P}_{i} \quad$ Minimum active power output of unit $i$.

$P_{i}^{\text {ini }} \quad$ Initial active power output of unit $i$.

$\bar{Q}_{i} \quad$ Reactive power capacity of unit $i$.

$\underline{Q}_{i} \quad$ Minimum reactive power output of unit $i$.

$R_{i}^{\mathrm{U}} \quad$ Maximum up-reserve that can be deployed by unit $i$.

$R_{i}^{\mathrm{D}} \quad$ Maximum down-reserve that can be deployed by unit $i$.

$R_{i}^{+} \quad$ Maximum Ramp-up rate of unit $i$.

$R_{i}^{-} \quad$ Maximum Ramp-down rate of unit $i$.

$r_{i}^{\text {ini }} \quad$ Initial reserve deployed by unit $i$.

$\bar{S}_{n m} \quad$ Capacity of transmission line $(n, m)$.

$u_{i}^{\text {ini }} \quad$ Initial commitment status of unit $i$.

$V_{d}^{\mathrm{SH}} \quad$ Value of load shed for load $d$.

$W_{k t s} \quad$ Wind power production realization of farm $k$ in period $t$ and scenario $s$.

$\bar{W}_{k t}^{\mathrm{DA}} \quad$ Maximum power production of wind farm $k$ in period $t$ that can be scheduled at the scheduling stage. It is assumed to be equal to the expected power production, i.e., $\bar{W}_{k t}^{\mathrm{DA}}=\sum_{s} \rho_{s} W_{k t s}, \forall k, \forall t$.

$x_{n m} \quad$ Reactance of transmission line $(n, m)$.

$Y_{n m} \quad$ Admittance magnitude of transmission line $(n, m)$.

$\phi_{n m} \quad$ Admittance angle of transmission line $(n, m)$.

6) Functions:

$P_{n m t}^{\mathrm{DA}}($.$) \quad Scheduled active power through transmission line$ $(n, m)$ in period $t$.

$P_{n m t s}($.$) \quad Active power through transmission line (n, m)$ in period $t$ and scenario $s$.

$Q_{n m t s}($.$) \quad Reactive power through transmission line (n, m)$ in period $t$ and scenario $s$.

$S_{n m t s}($.$) \quad Apparent power through transmission line (n, m)$ in period $t$ and scenario $s$.

The mathematical definitions of the functions above are given in Appendix A.

\section{INTRODUCTION}

\section{A. Motivation}

$\mathbf{U}$ NIT commitment (UC) is a crucial short-term decisionmaking problem in power system operations, whose objective is to determine the least-cost commitment and dispatch of generating units to serve the load. The deterministic form of the UC problem and its solution strategies are extensively documented in the literature, e.g., [1]-[3]. However, the recent increase of stochastic production units, especially wind power, in generation portfolios calls for a stochastic form of the UC problem, instead of a deterministic one. Moreover, a precise modeling of the physical laws characterizing this problem is needed as increasing wind power production generally results in stressed operating conditions. Hence, the need for an ac modeling arises.

\section{B. Literature Review and Contributions}

Large-scale integration of wind power increases significantly the level of uncertainty [4], hence the need of a stochastic UC approach. The stochastic UC problem was first studied in mid 1990s [5], [6]. More recent works include [7]-[9]. These approaches embed a dc representation of transmission system, rendering a mixed-integer linear UC problem (network-constrained dc-UC problem), which is generally tractable [10]-[13]. It is worth mentioning that due to the simplifications considered in the dc-UC problems, i.e., the exclusion of voltage magnitude and reactive power constraints, an ex-post verification is required to check that the results obtained are implementable.

A UC problem including an ac network representation (network-constrained ac-UC problem) provides a comparatively more precise description of power system operations, particularly as operating conditions become increasingly stressed due to increasing wind production. However, the ac unit commitment (ac-UC) problem is mixed-integer nonlinear, and thus hard to solve. In the technical literature, there are few works addressing the ac-UC problem. Reference [14] proposes an approach based on Benders' decomposition to solve an ac network-constrained hydrothermal scheduling problem. A security-constrained ac-UC problem is proposed in [15], whose objective is to minimize the system's operating cost while maintaining appropriate security. The solution strategy proposed in [15] is to decompose the original ac-UC problem into a master problem without enforcing the network constraints, and a subproblem to check the feasibility of the master solution from the network constraint point of view. Note that wind power uncertainty is not modeled either in [14] or [15]. Reference [16] formulates a security-constrained stochastic ac-UC problem under wind power uncertainty, and discusses potential solution techniques, but numerical results are not reported.

This paper proposes a network-constrained ac-UC problem in which the wind power uncertainty is characterized by a set of suitable scenarios. To cope with wind power uncertainty, a two-stage stochastic programming model is considered, whose first-stage represents the day-ahead market, and whose secondstage represents the real-time operating conditions involving wind power realizations.

Considering the context above, the contributions of this paper are fourfold:

1) To propose a two-stage network-constrained ac-UC problem for a system with significant wind power production, which is stochastic, mixed-integer, nonlinear and generally hard to solve. Note that a very limited number of works in the literature consider the ac representation of transmission network within the UC problem [17].

2) To decompose the proposed network-constrained ac-UC problem by scenario and time period, which ease the computational burden. To the best of our knowledge, none of the previous works proposes a similar approach. Some papers are available in the literature considering the stochastic UC problem and solving it by decomposition approaches [17]; however all of them consider dc network constraints, not the more complex ac ones. Moreover, to 


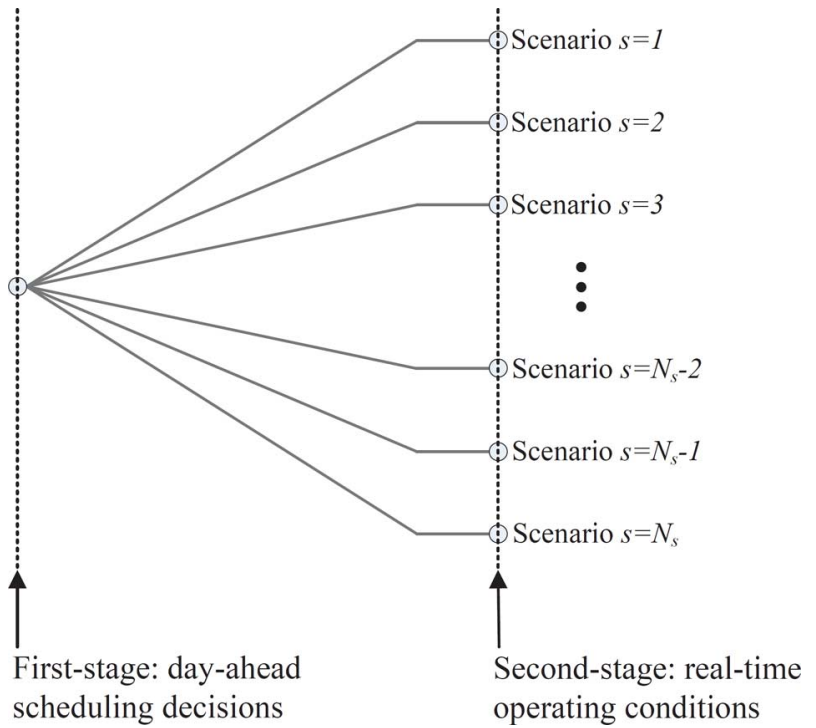

Fig. 1. Two-stage stochastic decision framework of the proposed ac-UC problem.

the best of our knowledge, no paper in the literature takes the advantages of wind uncertainty to convexify the ac-UC problem, making it possible the use of decomposition techniques.

3) To implement Benders' decomposition, which requires iteratively solving a) a mixed-integer linear master problem, and b) a set of nonlinear, but continuous subproblems.

4) To endogenously model voltage constraints within the proposed network-constrained ac-UC framework, and to numerically show that those constraints may result in a different commitment of generating units. This is important since such constraints are not enforced within the dc-UC problems, which are commonly used in practice.

Note that in the rest of this paper the terms "dc-UC" and "ac-UC" refer to "network-constrained dc-UC" and "networkconstrained ac-UC" problems, respectively.

\section{AC-UC MODEL}

\section{A. Stochastic Framework}

Fig. 1 depicts the two-stage stochastic framework considered in this paper. Note that these two stages are simultaneously considered. The first-stage includes the scheduling decisions made at the day-ahead market. Such decisions are adapted to any wind production realization in the second-stage, where the real-time operating conditions corresponding to individual wind scenarios are represented. This way, prior to the uncertainty realization in the second-stage, the system is optimally prepositioned via scheduling decisions in the first-stage. Therefore, the first-stage decisions are scenario-independent, while each second-stage decision adapts to the operating conditions of the corresponding wind power realization. Further details on the two-stage stochastic programming model used in this paper can be found in [4].

The proposed ac-UC problem clears the day-ahead market while considering all plausible real-time operating conditions. Therefore, the scheduling decisions made at the first-stage (day- ahead market) are consistent with those conditions. Since operating limits are enforced explicitly at the second-stage for all plausible operating conditions, the resulting schedule is both optimal and consistent with real-time operating conditions.

\section{B. Modeling Assumptions}

For the sake of clarity, the modeling assumptions considered in this work are listed as follows:

1) The first-stage of the proposed UC problem (that represents the day-ahead market) embodies a dc network representation, while the second-stage (that represents the real-time operation) embeds an ac one. This assumption is consistent with the functioning of most real-world electricity markets.

2) For the sake of simplicity, only wind power uncertainty is taken into account. However, other uncertainties can be incorporated into the model. The uncertainty of wind power production is modeled through a set of plausible scenarios based on the available forecasted data.

3) The minimum up-time and minimum down-time constraints of thermal units are not considered in this paper. To consider them, additional binary variables are required [18].

4) A number of units are available to provide reserve.

5) The wind power production cost is assumed to be nil.

6) All loads are assumed to be inelastic.

7) Wind farms of Type 3 DFIG and Type 4 full converter are able to provide voltage support in steady-state and dynamically [19]. However, for the sake of simplicity, we assume unit power factor for all wind farms.

8) The security constraints are not modeled in this work. However, such constraints can be easily incorporated in the proposed framework.

\section{Formulation}

The considered two-stage ac-UC problem is formulated as (1)-(3). Objective function (1) represents the system's expected cost, and is subject to first-stage constraints (2) and secondstage constraints (3). The optimization variables of the ac-UC problem (1)-(3) are the elements of the set:

$$
\begin{aligned}
& \Xi^{\mathrm{UC}}=\left\{\theta_{n t}^{\mathrm{DA}}, C_{i t}^{\mathrm{SU}}, P_{i t}^{\mathrm{DA}}, W_{k t}^{\mathrm{DA}}, u_{i t}, v_{n t s}, \theta_{n t s}, r_{i t s}, Q_{i t s},\right. \\
& \left.W_{k t s}^{\mathrm{SP}}, L_{d t s}^{\mathrm{SH}}\right\} \text {. }
\end{aligned}
$$

Note that the mathematical definitions of the functions used in (2) and (3) are given in Appendix A.

The objective function is

$$
\begin{aligned}
\underset{\Xi^{\mathrm{UC}}}{\operatorname{Minimize}} & \sum_{(i \in \mathcal{G}) t}\left[C_{i t}^{\mathrm{SU}}+C_{i} P_{i t}^{\mathrm{DA}}\right] \\
& +\sum_{s} \rho_{s}\left[\sum_{(i \in \mathcal{G}) t} C_{i} r_{i t s}+\sum_{(d \in \mathcal{D}) t} V_{d}^{\mathrm{SH}} L_{d t s}^{\mathrm{SH}}\right]
\end{aligned}
$$

The first two terms of (1) correspond to the system's cost at scheduling time (first-stage), while the other two terms refer to the expected cost in real-time operation (second-stage). The first term represents the start-up cost of the units, the second one refers to their production cost, and the third term represents the reserve deployment cost. Note that the reserve deployment cost 
(third term) refers to the production cost of the additional energy produced in real-time operation to offset the energy imbalance occurred due to wind power variability. This term is in fact the product of the generating unit's marginal cost and the production increment from day-ahead to real-time operation. Finally, the last term of (1) is the load curtailment cost.

The first-stage constraints are

$$
\begin{array}{ll}
\sum_{i \in \mathcal{G}_{n}} P_{i t}^{\mathrm{DA}}+\sum_{k \in \mathcal{K}_{n}} W_{k t}^{\mathrm{DA}}-\sum_{d \in \mathcal{D}_{n}} L_{d t}^{\mathrm{P}}=\sum_{m \in \Omega_{n}} P_{n m t}^{\mathrm{DA}}(\boldsymbol{\theta}) \\
\underline{P}_{i} u_{i t} \leq P_{i t}^{\mathrm{DA}} \leq \bar{P}_{i} u_{i t}, \forall t \\
0 \leq W_{k t}^{\mathrm{DA}} \leq \bar{W}_{k t}^{\mathrm{DA}} & \forall i \in \mathcal{G}, \forall t \\
-R_{i}^{-} \leq\left[P_{i(t=1)}^{\mathrm{DA}}-P_{i}^{\mathrm{ini}}\right] \leq R_{i}^{+} & \forall i \in \mathcal{G} \\
-R_{i}^{-} \leq\left[P_{i t}^{\mathrm{DA}}-P_{i(t-1)}^{\mathrm{DA}}\right] \leq R_{i}^{+} & \forall i \in \mathcal{G}, \forall t>1 \\
-\pi \leq \theta_{n t}^{\mathrm{DA}} \leq \pi & \forall n, \forall t \\
\theta_{(n=1) t}^{\mathrm{DA}}=0 & \forall t \\
P_{n m t}^{\mathrm{DA}}(\boldsymbol{\theta}) \leq \bar{S}_{n m} & \forall n, \forall m \in \Omega_{n}, \forall t \\
C_{i(t=1)}^{\mathrm{SU}} \geq \lambda_{i}^{\mathrm{SU}}\left[u_{i(t=1)}-u_{i}^{\mathrm{ini}}\right] & \forall i \in \mathcal{G} \\
C_{i t}^{\mathrm{SU}} \geq \lambda_{i}^{\mathrm{SU}}\left[u_{i t}-u_{i(t-1)}\right. & \forall i \in \mathcal{G}, \forall t>1 \\
C_{i t}^{\mathrm{SU}} \geq 0 & \forall i \in \mathcal{G}, \forall t .
\end{array}
$$

Constraints $(2 \mathrm{a})$ represent the active power balance at scheduling time at each node and for each time period. Constraints (2b) and (2c) enforce the lower and upper bounds for active power production of generating units and wind farms, respectively. Constraints $(2 \mathrm{~d})$ and $(2 \mathrm{e})$ ensure that the hourly changes of scheduled power do not violate the ramp-rate limits. Constraints (2f) enforce lower and upper bounds of voltage angles. Constraints ( $2 \mathrm{~g})$ set $n=1$ as the reference node. The capacity of each transmission line is enforced through $(2 \mathrm{~h})$. Constraints (2i)-(2k) allow calculating the start-up cost of the units.

The second-stage constraints are

$$
\begin{aligned}
& \sum_{i \in \mathcal{G}_{n}}\left(P_{i t}^{\mathrm{DA}}+r_{i t s}\right)+\sum_{k \in \mathcal{K}_{n}}\left(W_{k t s}-W_{k t s}^{\mathrm{SP}}\right) \\
& -\sum_{d \in \mathcal{D}_{n}}\left(L_{d t}^{\mathrm{P}}-L_{d t s}^{\mathrm{SH}}\right)=\sum_{m \in \Omega_{n}} P_{n m t s}(\mathbf{v}, \boldsymbol{\theta}) \forall n, \forall t, \forall s \\
& \sum_{i \in \mathcal{G}_{n}} Q_{i t s}-\sum_{d \in \mathcal{D}_{n}} L_{d t}^{\mathrm{Q}}=\sum_{m \in \Omega_{n}} Q_{n m t s}(\mathbf{v}, \boldsymbol{\theta}) \\
& \forall n, \forall t, \forall s \\
& 0 \leq L_{d t s}^{\mathrm{SH}} \leq L_{d t}^{\mathrm{P}} \\
& \forall d \in \mathcal{D}, \forall t, \forall s \\
& 0 \leq W_{k t s}^{\mathrm{SP}} \leq W_{k t s} \\
& \forall k \in \mathcal{K}, \forall t, \forall s \\
& -R_{i}^{\mathrm{D}} \leq r_{i t s} \leq R_{i}^{\mathrm{U}} \\
& \forall i \in \mathcal{G}, \forall t, \forall s \\
& \underline{P}_{i} u_{i t} \leq\left[r_{i t s}+P_{i t}^{\mathrm{DA}}\right] \leq \bar{P}_{i} u_{i t} \quad \forall i \in \mathcal{G}, \forall t, \forall s \\
& Q_{i} u_{i t} \leq Q_{i t s} \leq \bar{Q}_{i} u_{i t} \quad \forall i \in \mathcal{G}, \forall t, \forall s \\
& -R_{i}^{-} \leq\left[\left(P_{i(t=1)}^{\mathrm{DA}}+r_{i(t=1) s}\right)-\left(P_{i}^{\mathrm{ini}}+r_{i}^{\mathrm{ini}}\right)\right] \leq R_{i}^{+} \\
& \forall i \in \mathcal{G}, \forall s \\
& -R_{i}^{-} \leq\left[\left(P_{i t}^{\mathrm{DA}}+r_{i t s}\right)-\left(P_{i(t-1)}^{\mathrm{DA}}+r_{i(t-1) s}\right)\right] \leq R_{i}^{+} \\
& \forall i \in \mathcal{G}, \forall t>1, \forall s \\
& \underline{v}_{n} \leq v_{n t s} \leq \bar{v}_{n} \\
& \forall n, \forall t, \forall s
\end{aligned}
$$

$$
\begin{array}{lr}
-\pi \leq \theta_{n t s} \leq \pi & \forall n, \forall t, \forall s \\
\theta_{(n=1) t s}=0 & \forall t, \forall s \\
S_{n m t s}(\mathbf{v}, \boldsymbol{\theta}) \leq \bar{S}_{n m} & \forall n, \forall m \in \Omega_{n}, \forall t, \forall s .
\end{array}
$$

Constraints (3a) and (3b) represent the active and reactive power balance in real-time operation at each node and for each time period and scenario. Active power balance constraints (3a) enforce that the deviations of wind production are met with reserve deployment of generating units, and/or wind power spillage of farms, and/or curtailment of loads. Constraints (3c)-(3g) bound the value of unserved load, wind power spillage, active power reserve deployed, total active power production and reactive power production of generating units, respectively. Constraints (3h) and (3i) enforce ramp-rate limits. Constraints $(3 \mathrm{j})$ enforce the lower and upper bounds of nodes' voltage magnitude. Finally, constraints $(3 \mathrm{k})-(3 \mathrm{~m})$ are similar to $(2 \mathrm{f})-(2 \mathrm{~h})$, but for real-time operation.

Finally, note that the proposed ac-UC problem (1)-(3) is mixed-integer, nonlinear and generally intractable. To make it solvable, Benders' decomposition is applied as described in the next section.

\section{BENDERS' SOLUTION}

This section proposes a solution strategy based on Benders' decomposition to solve problem (1)-(3).

\section{A. Complicating Variables and Convexification}

If first-stage variables $P_{i t}^{\mathrm{DA}}$ and $u_{i t}$ are fixed to given values in problem (1)-(3), this problem decomposes into 1) a scenario-independent mixed-integer linear problem (representing the first-stage), and 2) a set of nonlinear continuous problems, one per scenario (representing the second-stage). Therefore, $P_{i t}^{\mathrm{DA}}$ and $u_{i t}$ are complicating variables, and Benders' decomposition can be potentially applied [20].

Although the original ac-UC problem (1)-(3) is non-convex and Benders' decomposition is not generally applicable, if the number of wind power scenarios is large enough, the objective function (1) as a function of the complicating variables convexifies as shown in [21]. In other words, the objective function of an expected value stochastic programming problem convexifies as the number of scenarios increases. The reason of this is that the objective function represents the expectation over a number of scenarios. Thus, as the number of scenarios increases, the diversity of objective functions increases, while the weight of each single-scenario decreases. This results in a smoothing effect leading to the convexification of the expected value objective function. This convexification allows a successful implementation of Benders' decomposition.

Benders' convergence is guaranteed if the objective function of the original problem projected on the subspace of the complicating variables has a convex envelope. The proposed ac-UC problem (1)-(3) is "sufficiently" convexified by considering a large enough number of scenarios, and our numerical analysis confirms the well-functioning of the proposed decomposition algorithm. Nevertheless, convergence cannot be generally guaranteed for the considered problem.

Finally, note that the asymptotic convexification yielded by increasing the number of scenarios is not a heuristic, provided 
that a large-enough number of scenarios is considered. As the number of scenarios increases, the objective function of the considered model asymptotically convexifies. Thus, it seems reasonable to approximate the objective function with a convex objective.

\section{B. Decomposition by Scenario and Time Period}

Fixing the complicating variables $P_{i t}^{\mathrm{DA}}$ and $u_{i t}$ to given values decomposes the ac-UC problem (1)-(3) by scenario. However, the ramping constraints (3h) and (3i), which links time periods, impede the ac-UC problem to decompose by time period.

In general, an appropriate balance is needed between model accuracy and computational burden. To this end, a heuristic technique is used in this paper to decompose the proposed ac-UC problem by time period. This technique allows reducing the computational burden, but at the potential cost of introducing imprecision in the final solution. According to this heuristic technique, the inter-temporal ramping constraints (3h) and (3i) are relaxed and enforced just locally. That is, at time $t$, ramping limits are enforced with respect to time $t-1$, and time periods are processed successively from the first to the last one. However, note that if needed, a reduced number of hours (e.g., 3 or 4) may be processed at the same time, which may be helpful for periods with high increase/decrease in demand and/or renewable production levels. Our extensive numerical simulations show that the results obtained with and without such a heuristic technique are close enough. Note that the temporal decomposition used is myopic, and it cannot be applied in power systems with inter-temporal constraints (e.g., a power system with a significant number of hydro units or large-scale energy storage facilities) since these inter-temporal constraints cannot be locally relaxed. Hydroelectric systems are not prevalent in most parts of the world [22], but for such systems the proposed ac-UC problem is still applicable and computationally efficient by decomposing the problem only by scenario (and not by time period). Note that the number of scenarios is generally much larger than the number of hours within the time horizon considered.

The formulation of Benders' master problem and subproblems are provided in the next subsections.

\section{Subproblem}

The subproblem for scenario $s$ and time period $t$ is formulated as (4) below. All variables pertain to Benders' iteration $\nu$ :

$$
\begin{aligned}
&\left\{\underset{\Xi_{t s}^{\mathrm{SP}}}{\operatorname{Minimize}} Z_{t s}^{(\nu)}=\sum_{i \in \mathcal{G}} C_{i} r_{i t s}^{(\nu)}+\sum_{d \in \mathcal{D}} V_{d}^{\mathrm{SH}} L_{d t s}^{\mathrm{SH}^{(\nu)}}\right. \\
& \text { subject to } \\
&(3) \forall i \in \mathcal{G} \\
& P_{i t}^{\mathrm{DA}}{ }^{(\nu)}=P_{i t}^{\mathrm{DA}, \text { fixed }} \forall i \in \mathcal{G} \\
& u_{i t}^{(\nu)}=u_{i t}^{\text {fixed }}\} \forall t, \forall s .
\end{aligned}
$$

Objective function (4a) represents total operation costs in real time operation. Constraint (4b) comprises the second-stage constraints. Constraints (4c) and (4d) fix the values of the complicating variables to given values obtained from the solution of the master problem. The formulation of the master problem is provided in the next subsection.

Reactive power constraints (3g) and voltage magnitude constraints (3j) are enforced in each subproblem (4), while similar constraints are not enforced in the master problem. Thus, the fixed values in (4c) and (4d) obtained from the master problem may make subproblems (4) infeasible. To prevent infeasibility, a number of non-negative slack variables are included in the reactive power and voltage magnitude constraints, along with penalties in the objective function (4a) [20], [23]. Thus, the always-feasible form of (4) is (5) as follows:

$$
\begin{aligned}
& \left\{\underset{\Xi_{t s}^{\mathrm{SP}}}{\operatorname{Minimize}} Z_{t s}^{(\nu)}=\sum_{i \in \mathcal{G}} C_{i} r_{i t s}^{(\nu)}+\sum_{d \in \mathcal{D}} V_{d}^{\mathrm{SH}} L_{d t s}^{\mathrm{SH}^{(\nu)}}\right. \\
& +\sum_{i \in \mathcal{G}} h^{\mathrm{Q}}\left(\underline{Q}_{i t s}^{\mathrm{aux}^{(\nu)}}+\bar{Q}_{i t s}^{\mathrm{aux}^{(\nu)}}\right) \\
& +\sum_{n} h^{\mathrm{v}}\left(\underline{v}_{n t s}^{\mathrm{aux}^{(\nu)}}+\bar{v}_{n t s}^{\mathrm{aux}^{(\nu)}}\right) \\
& (3 \mathrm{a})-(3 \mathrm{f}),(3 \mathrm{~h}),(3 \mathrm{i}),(3 \mathrm{k})-(3 \mathrm{~m}) \\
& \left(\underline{Q}_{i}-\underline{Q}_{i t s}^{\mathrm{aux}^{(\nu)}}\right) u_{i t} \leq Q_{i t s}^{(\nu)} \leq\left(\bar{Q}_{i}+\bar{Q}_{i t s}^{\mathrm{aux}^{(\nu)}}\right) u_{i t} \\
& \forall i \in \mathcal{G}(5 \mathrm{c}) \\
& \left(\underline{v}_{n}-\underline{v}_{n t s}^{\mathrm{aux}^{(\nu)}}\right) \leq v_{n t s}^{(\nu)} \leq\left(\bar{v}_{n}+\bar{v}_{n t s}^{\mathrm{aux}^{(\nu)}}\right) \quad \forall n \\
& \underline{Q}_{i t s}^{\operatorname{aux}^{(\nu)}} \geq 0, \bar{Q}_{i t s}^{\operatorname{aux}^{(\nu)}} \geq 0 \\
& \forall i \in \mathcal{G}(5 \mathrm{e}) \\
& \underline{v}_{n t s}^{\operatorname{aux}^{(\nu)}} \geq 0, \bar{v}_{n t s}^{\operatorname{aux}^{(\nu)}} \geq 0 \\
& P_{i t}^{\mathrm{DA}^{(\nu)}}=P_{i t}^{\mathrm{DA}, \text { fixed }} \\
& u_{i t}^{(\nu)}=u_{i t}^{\text {fixed }} \\
& : \mu_{i t s}^{\mathrm{P}^{(\nu)}} \\
& \forall n \\
& \forall i \in \mathcal{G}(5 \mathrm{~g}) \\
& : \mu_{i t s}^{\mathrm{u}^{(\nu)}} \\
& \forall i \in \mathcal{G} \text { (5h) } \\
& \} \quad \forall t, \forall s \text {. }
\end{aligned}
$$

The optimization variables pertaining to each subproblem (5) are the elements of the set:

$$
\begin{array}{r}
\Xi_{t s}^{\mathrm{SP}}=\left\{Z_{t s}^{(\nu)}, P_{i t}^{\mathrm{DA}}{ }^{(\nu)}, u_{i t}^{(\nu)}, v_{n t s}^{(\nu)}, \theta_{n t s}^{(\nu)}, r_{i t s}^{(\nu)}, Q_{i t s}^{(\nu)}, W_{k t s}^{\mathrm{SP}^{(\nu)}}\right. \\
\left.L_{d t s}^{\mathrm{SH}^{(\nu)}}, \underline{Q}_{i t s}^{\mathrm{aux}^{(\nu)}}, \bar{Q}_{i t s}^{\operatorname{aux}^{(\nu)}}, \underline{v}_{n t s}^{\mathrm{aux}^{(\nu)}}, \bar{v}_{n t s}^{\operatorname{aux}^{(\nu)}}\right\} .
\end{array}
$$

Note that $\underline{Q}_{i t s}^{\mathrm{aux}^{(\nu)}}, \bar{Q}_{i t s}^{\mathrm{aux}}{ }^{(\nu)}, \underline{v}_{n t s}^{\mathrm{aux}}{ }^{(\nu)}$ and $\bar{v}_{n t s}^{\mathrm{aux}^{(\nu)}}$ are non-negative slack variables, while $h^{\mathrm{Q}}$ and $h^{\mathrm{v}}$ are large enough positive constants.

Note also that $u_{i t}$ is a continuous variable in each subproblem (5), while it is a binary variable in the original problem (1)-(3) and the master problem (7). Thus, each subproblem (5) is continuous and nonlinear.

The complicating variables are fixed through constraints $(5 \mathrm{~g})$ and (5h), whose dual variables, $\mu_{i t s}^{\mathrm{P}^{(\nu)}}$ and $\mu_{i t s}^{\mathrm{u}^{(\nu)}}$, provide sensitivities to be used in building Benders' cuts for the master problem. These sensitivities are obtained as follows:

$$
\begin{array}{rlrl}
\mu_{i t}^{\mathrm{P}^{(\nu)}} & =\sum_{s} \rho_{s} \mu_{i t s}^{\mathrm{P}^{(\nu)}} & & \forall i \in \mathcal{G}, \forall t \\
\mu_{i t}^{\mathrm{u}^{(\nu)}}=\sum_{s} \rho_{s} \mu_{i t s}^{\mathrm{u}^{(\nu)}} & \forall i \in \mathcal{G}, \forall t .
\end{array}
$$


In addition, the following value is calculated to be used in (6d) and master problem (7):

$$
Z^{(\nu)}=\sum_{t s} \rho_{s} Z_{t s}^{(\nu)}
$$

The upper bound for the optimal value of objective function of the original problem (1)-(3) at iteration $\nu$ is obtained as

$$
Z_{u p}^{(\nu)}=Z^{(\nu)}+\sum_{(i \in \mathcal{G}) t}\left[C_{i t}^{\mathrm{SU}, \text { fixed }}+C_{i} P_{i t}^{\mathrm{DA}, \mathrm{fixed}}\right]
$$

where the value of $C_{i t}^{\mathrm{SU} \text {,fixed }}$ is calculated using fixed values for $u_{i t}^{\text {fixed }}$.

\section{Master Problem}

The master problem corresponding to the original problem (1) - (3) is formulated as (7) below. All variables refer to Benders' iteration $\nu$. The objective function (7a) corresponds to (1), where $\alpha^{(\nu)}$ represents the expected cost in real-time operation:

$$
\begin{aligned}
& \underset{\Xi^{\mathrm{MP}}}{\operatorname{Minimize}} Z_{\text {down }}^{(\nu)}=\sum_{(i \in \mathcal{G}) t}\left[C_{i t}^{\mathrm{SU}^{(\nu)}}+C_{i} P_{i t}^{\mathrm{DA}^{(\nu)}}\right]+\alpha^{(\nu)}(7 \mathrm{a}) \\
& \text { subject to : } \\
& Z^{(j)}+\sum_{(i \in \mathcal{G}) t} \mu_{i t}^{\mathrm{P}^{(j)}}\left(P_{i t}^{\mathrm{DA}^{(\nu)}}-P_{i t}^{\mathrm{DA}^{(\mathrm{j})}}\right) \\
& +\sum_{(i \in \mathcal{G}) t} \mu_{i t}^{\mathrm{u}^{(j)}}\left(u_{i t}^{(\nu)}-u_{i t}^{(j)}\right) \leq \alpha^{(\nu)} j=1, \ldots, \nu-1 \quad(7 \mathrm{~b}) \\
& \alpha^{(\nu)} \geq \alpha^{\text {down }} \\
& (2 \mathrm{a}),(2 \mathrm{c})-(2 \mathrm{k}) \\
& P_{i t}^{\mathrm{DA}^{(\nu)}}+\left[\max _{s} r_{i t s}^{(\nu-1)}\right] u_{i t}^{(\nu)} \leq \bar{P}_{i} u_{i t}^{(\nu)} \quad \forall i \in \mathcal{G}, \forall t(7 \mathrm{e}) \\
& P_{i t}^{\mathrm{DA}^{(\nu)}}+\left[\min _{s} r_{i t s}^{(\nu-1)}\right] u_{i t}^{(\nu)} \geq \underline{P}_{i} u_{i t}^{(\nu)} \quad \forall i \in \mathcal{G}, \forall t . \quad(7 \mathrm{f})
\end{aligned}
$$

Master problem (7) is mixed-integer linear, and its optimization variables are the elements of the set:

$$
\Xi^{\mathrm{MP}}=\left\{Z_{\text {down }}^{(\nu)}, \theta_{n t}^{\mathrm{DA}^{(\nu)}}, C_{i t}^{\mathrm{SU}^{(\nu)}}, P_{i t}^{\mathrm{DA}^{(\nu)}}, W_{k t}^{\mathrm{DA}^{(\nu)}}, u_{i t}^{(\nu)}, \alpha^{(\nu)}\right\} .
$$

Constraints ( $7 \mathrm{~b})$ are Benders' cuts, which are generated one per iteration. Note that the feasibility cuts are not required in the master problem because always-feasible subproblems (5) are used within the proposed Benders' algorithm. This "trick" has proven to be computationally efficient. Constraint (7c) imposes a lower bound on $\alpha^{(\nu)}$ to accelerate convergence. Constraint (7d) enforce all first-stage constraints, except (2b). Instead of (2b), constraints (7e) and (7f) are included in the master problem to improve convergence. Note that $\max _{s} r_{i t s}^{(\nu-1)}$ and $\min _{s} r_{i t s}^{(\nu-1)}$ are parameters obtained from the solution of the subproblems in the previous iteration. In fact, in addition to the Benders' cuts (7b), constraints (7e) and (7f) further link the master problem and the subproblems.

The value of objective function (7a), i.e., $Z_{\text {down }}^{(\nu)}$, is a lower bound for the optimal value of the objective function of problem (1)-(3). The solution of the master problem (7) updates the values of complicating variables.

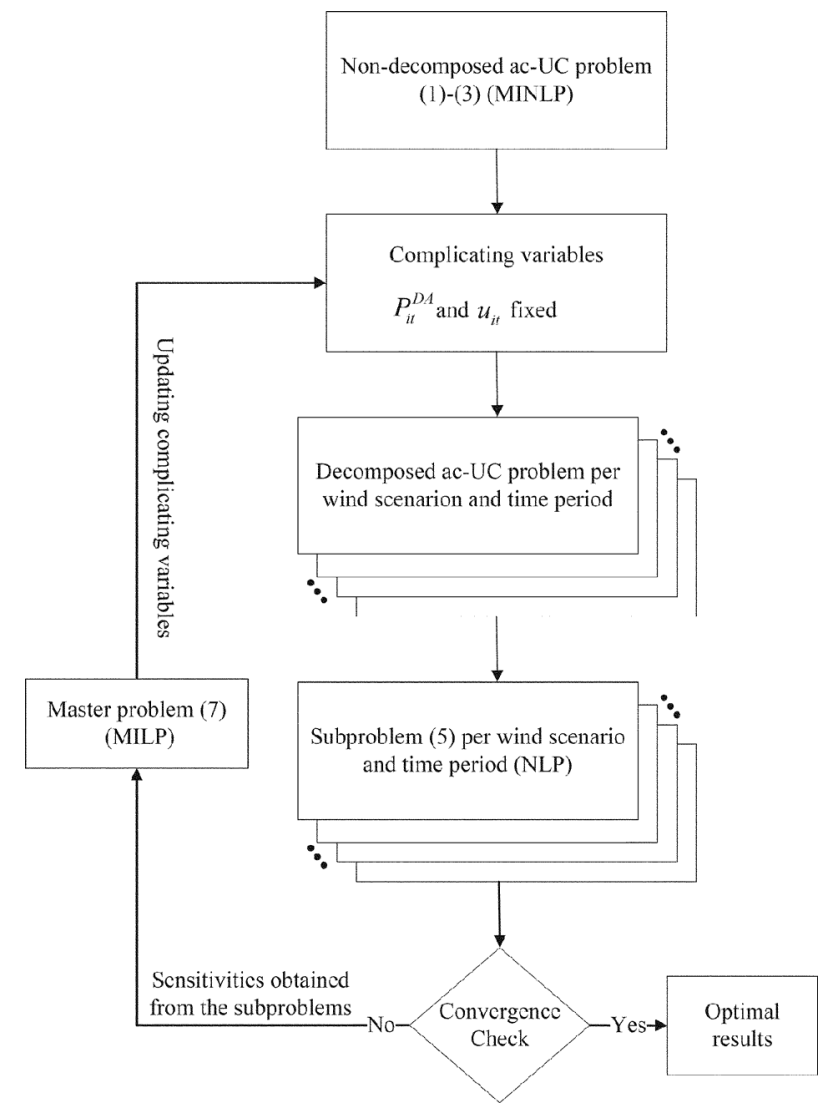

Fig. 2. Flowchart of the proposed Benders' algorithm.

\section{E. Benders' Algorithm}

The proposed Benders' algorithm is as follows:

1) Input: a small tolerance $\varepsilon$ to control convergence, and initial guesses of the complicating variables, $P_{i t}^{\mathrm{DA}^{0}}$ and $u_{i t}^{0} \forall i \in \mathcal{G}, \forall t$.

2) Initialization: Set $\nu=1, Z_{\text {down }}^{(\nu)}=-\infty$, $P_{i t}^{\mathrm{DA}, \text { fixed }}=P_{i t}^{\mathrm{DA}^{0}}$ and $u_{i t}^{\mathrm{fixed}}=u_{i t}^{0} \forall i \in \mathcal{G}, \forall t$.

3) Initial scenario: Consider scenario $s=1$.

4) Initial time period: Consider time period $t=1$.

5) Subproblem solution: Solve (5) for scenario $s$ and time period $t$ and calculate $Z_{t s}^{(\nu)}$.

6) Next time period: Consider the next time period, and repeat step 5. If all time periods have been considered, go to the next step.

7) Next scenario: Consider the next scenario, and repeat steps 4 to 6 . If all scenarios have been considered, go to the next step.

8) Convergency check: If $\left|Z_{u p}^{(\nu)}-Z_{\text {down }}^{(\nu)}\right| \leq \varepsilon$, the optimal solution with a level of accuracy $\varepsilon$ has been obtained. Otherwise, calculate $Z^{(\nu)}$ and the sensitivities to build the next Benders' cut. Then, set $\nu \longleftarrow \nu+1$.

9) Master problem solution: Solve (7), calculate $Z_{\text {down }}^{(\nu)}$ and update the values of complicating variables. Then, continue in step 3.

The flowchart of the proposed Benders' algorithm is depicted in Fig. 2. 
TABLE I

NETWORK DATA

\begin{tabular}{c|c|c|c|c|c|c}
\hline Transmission line $(n, m)$ & $1-3$ & $3-9$ & $6-10$ & $10-11$ & $11-14$ & $14-16$ \\
\hline $\bar{S}_{n m}$ [p.u.] & 2.3 & 1.6 & 2.5 & 3.0 & 2.3 & 2.1 \\
\hline
\end{tabular}

TABLE II

DATA FOR GENERATING UNITS

\begin{tabular}{|c|c|c|c|c|c|c|c|c|c|c|c|c|}
\hline & & $\underline{P}_{i}$ & $\bar{P}_{i}$ & $\underline{Q}_{i}$ & $\bar{Q}_{i}$ & $\begin{array}{c}R_{i}^{\mathrm{U}}, \\
R_{i}^{\mathrm{D}} \\
{[\text { p.u.] }}\end{array}$ & {$\left[\begin{array}{l}R_{i}^{+}, \\
R_{i}^{-} \\
{[\text {p.u. }}\end{array}\right.$} & $\begin{array}{l}C_{i} \\
{\left[\frac{\Phi}{\mathrm{p} \cdot \mathrm{u}}\right.}\end{array}$ & $\lambda_{i}^{\mathrm{SU}}$ & $u_{i}^{\mathrm{in} \mathrm{i}}$ & $P_{i}^{\mathrm{ini}}$ & \\
\hline & 1 & & & 0.00 & 0.10 & & & & 200 & & & \\
\hline & 1 & & & -0.25 & 0.30 & & 0.500 & 246 & & & & \\
\hline 5,6 & 2 & & 00 & 0.00 & 10 & 000 & .100 & 09 & 30 & & & \\
\hline 7,8 & 2 & & 760 & .25 & 030 & 100 & 0.500 & 246 & 40 & & & \\
\hline & 7 & & & & & & 2500 & & & & & \\
\hline & & & & & & & & & & & & \\
\hline & & & & & & 450 & 1.150 & 1408 & 500 & & & \\
\hline-19 & 17 & 24 & 20 & & 06 & 096 & 0.096 & 2141 & 40 & & & \\
\hline 20 & 1 & 500 & 550 & .50 & 0.80 & 0.450 & 1.000 & 1592 & 20 & & 1 & 0.4 \\
\hline 21 & 16 & 500 & 1.550 & -0.50 & 0.80 & 0.450 & 1.000 & 1592 & 20 & & .10 & \\
\hline 22 & & 000 & 4.000 & -050 & & 1.500 & 2800 & 1917 & 25 & & & \\
\hline 23 & & 1.000 & 4.000 & & & & 2.800 & & & & & \\
\hline-29 & & 000 & 0.500 & -0.10 & 0.16 & 0.150 & 0.300 & & 100 & & 0.50 & \\
\hline, 31 & 2. & 500 & 1.550 & -0.50 & 0.80 & 0.450 & 1.000 & 1592 & 200 & & 1.10 & 0.4 \\
\hline 32 & 23 & 800 & 3.500 & -0.25 & 1.50 & 1.000 & 2.500 & 1720 & 100 & & 0.00 & 0.0 \\
\hline
\end{tabular}

TABLE III

LOAD FACTOR CORRESPONDING TO EACH TIME PERIOD

\begin{tabular}{l|l|l|l|l|l|l|l|l|l|l|l|l}
\hline Time period & $t_{1}$ & $t_{2}$ & $t_{3}$ & $t_{4}$ & $t_{5}$ & $t_{6}$ & $t_{7}$ & $t_{8}$ & $t_{9}$ & $t_{10}$ & $t_{11}$ & $t_{12}$ \\
\hline
\end{tabular} \begin{tabular}{l|l|l|l|l|l|l|l|l|l|l|l|l}
\hline Load factor & 0.75 & 0.70 & 0.65 & 0.60 & 0.62 & 0.63 & 0.65 & 0.68 & 0.70 & 0.72 & 0.75 & 0.78
\end{tabular}

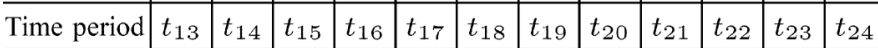
\begin{tabular}{l|l|l|l|l|l|l|l|l|l|l|l|l} 
Load factor & 0.80 & 0.85 & 0.85 & 0.90 & 0.92 & 0.95 & 0.98 & 1.00 & 0.97 & 0.93 & 0.91 & 0.92 \\
\hline
\end{tabular}

\section{CAse Study}

This section presents numerical results for a case study based on the IEEE one-area 24-node reliability test system (RTS) [24]. In this study, a daily time horizon (time periods $t_{1}$ to $t_{24}$ ) is considered. Under the per-unit system used, 1 p.u. of power is equivalent to $100 \mathrm{MW}$.

\section{A. Data}

Network data $\bar{S}_{n m}$ are those reported in [24], except the ones given in Table I. In addition, data for generating units are given in Table II. Note that generating units 1, 2, 5 and 6 do not provide reserve. Note also that to highlight voltage issues, the synchronous condenser connected to node 14 in [24] is removed.

Hourly active and reactive loads are those given in [24] multiplied by the hourly load factors provided in Table III. Accordingly, time period $t_{20}$ is the peak hour.

Two wind farms located at nodes 3 and 14 are considered, whose installed capacities are 2.85 p.u. and 2.96 p.u., respectively. Total wind capacity (i.e., 5.81 p.u.) is $13.71 \%$ of the total installed capacity ( 42.36 p.u.). The wind power production uncertainties are characterized through 40 scenarios, as illustrated

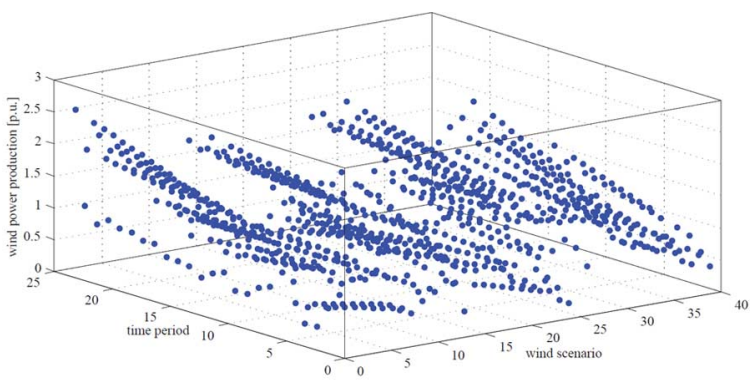

(a)

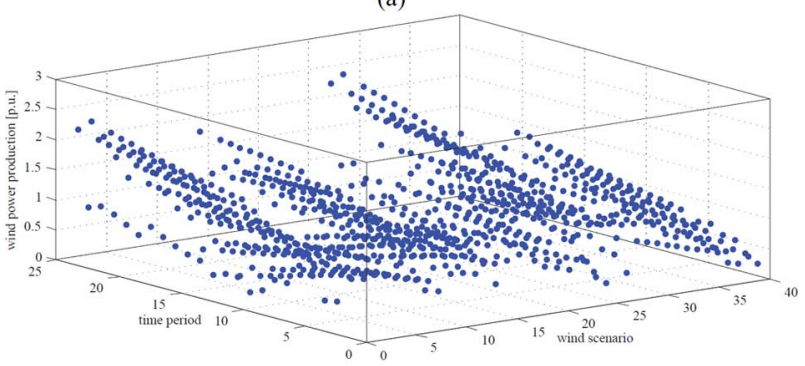

(b)

Fig. 3. Wind power scenarios of the farm (a) located at node 3, (b) and of that located at node 14 .

TABLE IV

Probability of EACH Scenario $\left(\rho_{s}\right)$

\begin{tabular}{c|c|c|c|c|c|c|c|c|c|c|c|c|c|c|c}
\hline$s$ & $\rho_{s}$ & $s$ & $\rho_{s}$ & $s$ & $\rho_{s}$ & $s$ & $\rho_{s}$ & $s$ & $\rho_{s}$ & $s$ & $\rho_{s}$ & $s$ & $\rho_{s}$ & $s$ & $\rho_{s}$ \\
\hline$s_{1}$ & 0.01 & $s_{6}$ & 0.03 & $s_{11}$ & 0.05 & $s_{16}$ & 0.01 & $s_{21}$ & 0.03 & $s_{26}$ & 0.01 & $s_{31}$ & 0.02 & $s_{36}$ & 0.03 \\
\hline$s_{2}$ & 0.01 & $s_{7}$ & 0.03 & $s_{12}$ & 0.05 & $s_{17}$ & 0.01 & $s_{22}$ & 0.04 & $s_{27}$ & 0.01 & $s_{32}$ & 0.02 & $s_{37}$ & 0.03 \\
\hline$s_{3}$ & 0.01 & $s_{8}$ & 0.03 & $s_{13}$ & 0.01 & $s_{18}$ & 0.02 & $s_{23}$ & 0.04 & $s_{28}$ & 0.01 & $s_{33}$ & 0.02 & $s_{38}$ & 0.04 \\
\hline$s_{4}$ & 0.02 & $s_{9}$ & 0.04 & $s_{14}$ & 0.01 & $s_{19}$ & 0.02 & $s_{24}$ & 0.05 & $s_{29}$ & 0.01 & $s_{34}$ & 0.02 & $s_{39}$ & 0.04 \\
\hline$s_{5}$ & 0.02 & $s_{10}$ & 0.04 & $s_{15}$ & 0.01 & $s_{20}$ & 0.03 & $s_{25}$ & 0.05 & $s_{30}$ & 0.01 & $s_{35}$ & 0.02 & $s_{40}$ & 0.04 \\
\hline
\end{tabular}

in Fig. 3, whose upper plot corresponds to the farm located at node 3 , and whose lower plot refers to the farm located at node 14. Note that each dot illustrates the wind production level of the corresponding farm for a given time period under a particular scenario. In other words, each scenario of any of the two plots contains 24 dots indicating the wind production levels of the corresponding farm over 24 time periods. These 40 scenarios have been derived from historical data, and accurately represent the uncertainty without making the considered problem computationally intractable. The corresponding probabilities $\left(\rho_{s}\right)$ are given in Table IV. Based on the scenarios considered, the expected total wind power production over the 24 time periods is 1.72 p.u. (i.e., $29.60 \%$ of the total wind capacity).

The value of load shedding for all demands $\left(V_{d}^{\mathrm{SH}}\right)$ is considered $10000 \$ /$ p.u. Finally, tolerance $\varepsilon$ required for convergence check is set to $0.3 \%$ of the value of the objective function (expected cost).

\section{B. Cases Considered}

Three different cases are analyzed:

Case A) This case refers to a dc-UC problem, where both stages embody a dc representation of the network. This problem is directly solved using a mixed-integer linear solver. 
This article has been accepted for inclusion in a future issue of this journal. Content is final as presented, with the exception of pagination.

TABLE V

Mathematical Characteristics of EaCh UC PRoblem

\begin{tabular}{c|c|c|c|c|c}
\hline Problem & Formulation & Type of optimization model & Binary variables included? & Available solver & Case \\
\hline Non-decomposed dc-UC & Combination of (1), (2), and (9) & MILP $^{a}$ & Yes & CPLEX [25] & A \\
\hline Non-decomposed ac-UC & Combination of (1), (2), and (3) & MINLP $^{b}$ & Yes & No off-the-shelf solver & - \\
\hline \multirow{2}{*}{$\begin{array}{c}\text { Decomposed } \\
\text { ac-UC }\end{array}$} & $\begin{array}{c}\text { Benders' algorithm } \\
\text { (depicted in Fig. 2) }\end{array}$ & $\begin{array}{c}\text { NLP }^{c} \\
\text { (subproblems) }\end{array}$ & No & CONOPT & {$[26]$} \\
\cline { 3 - 6 } & $\begin{array}{c}\text { including subproblems (5) } \\
\text { and master problem (7) }\end{array}$ & $\begin{array}{c}\text { MILP } \\
\text { (master problem) }\end{array}$ & Yes & CPLEX \\
{$[25]$} & \\
\hline
\end{tabular}

${ }^{a}$ Mixed-integer linear programming

${ }^{b}$ Mixed-integer non-linear programming

${ }^{c}$ Non-linear programming (continuous)

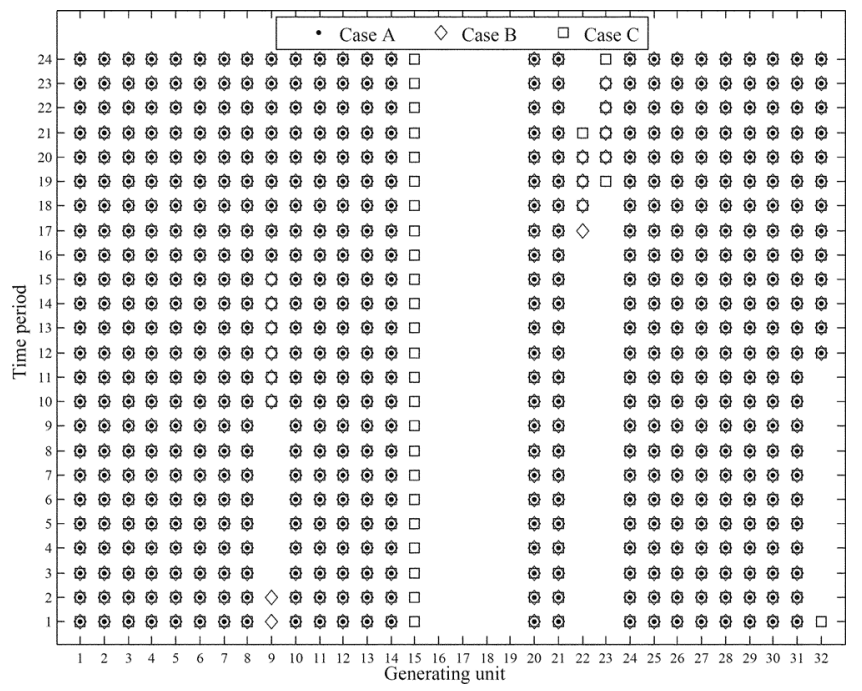

Fig. 4. Commitment status of the units for Cases A-C.

Case B) This case refers to an ac-UC problem based on model (1)-(3) solved by the proposed Benders' algorithm. The voltage magnitude of each node is enforced to be within 0.9 p.u. and 1.1 p.u.

Case C) This case is similar to Case B, but constraints on the voltage magnitude of nodes are relaxed. In this case, the voltage magnitude of nodes can lie within 0.5 p.u. and 1.5 p.u. Although this case is not realistic, it is considered for illustrative purposes.

Table $\mathrm{V}$ mathematically characterizes the non-decomposed dc-UC problem (Case A), the non-decomposed ac-UC problem, and the decomposed ac-UC problem (Cases B and C). Note that the non-decomposed dc-UC problem (Case A) includes the dc version of ac constraints (3), which is given in Appendix B as constraints (9).

The corresponding numerical results are given and analyzed in the next subsection.

\section{Numerical Results}

The expected cost, i.e., the value of objective function (1), for the three cases considered are given in the second column of Table VI. Additionally, the commitment status of the units are depicted in Fig. 4. Note that for all cases, neither load curtailment nor wind power spillage occurs.
TABLE VI

Numerical Results for CASES A-C

\begin{tabular}{l|c|c}
\hline & Expected cost [\$] & CPU time [minute] \\
\hline Case A & 638537.8 & 70 \\
\hline Case B & 651909.9 & 194 \\
\hline Case C & 650368.9 & 159 \\
\hline
\end{tabular}

According to Table VI and Fig. 4, the following observations are in order:

1) The expected cost in Case A (dc-UC problem) is comparatively smaller than that of Cases $\mathrm{B}$ and $\mathrm{C}$ (ac-UC problems). The reason of this is that the ac-UC problem enforces more realistic constraints than those of the dc-UC one. Also, the expected cost of Case B is comparatively higher than that of Case $\mathrm{C}$, where voltage magnitude constraints are not tightly enforced.

2) The dc-UC and ac-UC problems yield different commitment status for the generating units. According to Fig. 4, generating units 22 and 23 are decommitted in Case A (dc-UC problem), while they are committed in peak hours of Case B (ac-UC problem). Similarly, generating unit 9 is committed in time periods 1 and 2 of Case B.

3) In addition to the commitment status of generating units, the dispatch results of committed units from the dc-UC and ac-UC formulations are different. For example in Cases A and $\mathrm{B}$, generating unit 32 is scheduled in peak hour $t_{20} 3.2$ p.u. and 2.5 p.u., respectively.

4) The reason for these differences is the constraints that are not modeled in the dc-UC formulation (Case A), i.e., reactive power constraints (3g) and voltage magnitude constraints (3j). For example, the numerical results of Case B (ac-UC problem) illustrate that the voltage magnitudes of node 6 within different scenarios and time periods are mostly equal to its upper bound, i.e., 1.1 p.u. However, the results obtained by dc-UC model (Case A) misrepresent such constraint, since it does not consider the voltage magnitude bounds. In addition to constraints $(3 \mathrm{~g})$ and $(3 \mathrm{j})$, power losses in the network is the other reason of differences, as it is modeled in the ac-UC problem (Case B), but it is not in the dc-UC problem (Case A).

5) According to Fig. 4, although both Cases B and C solve ac-UC problems, different bounds on voltage magnitude 


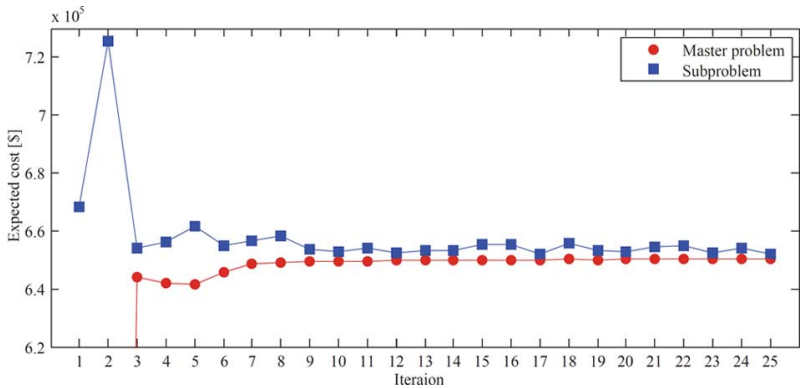

Fig. 5. Evolution of Benders' algorithm in Case B.

constraints $(3 \mathrm{j})$ render slightly different results. For example, the comparatively small generating unit 15 is fully committed in Case C, while it is fully decommitted in Case B. On the other hand, the comparatively large generating unit 9 is not committed in Case $\mathrm{C}$ for time periods $t_{1}$ and $t_{2}$, while it is in Case B. These differences allow concluding that the voltage magnitude constraint may potentially alter the commitment results.

Finally, Fig. 5 illustrates the evolution of Benders' algorithm in Case B (ac-UC problem). This algorithm converges in iteration 25 , where the difference between $Z_{u p}^{(\nu)}$ (upper curve) and $Z_{\text {down }}^{(\nu)}$ (lower curve) is smaller than the considered tolerance.

\section{Computational, Optimality, and Implementation Issues}

Each subproblem (5) is solved using CONOPT [26] under GAMS [27], while the master problem (7) is solved using CPLEX 12.1 [25] under GAMS [27], both on a Sun Fire $\mathrm{X} 4600 \mathrm{M} 2$ with 8 Quad-Core processors clocking at $2.9 \mathrm{GHz}$ and $256 \mathrm{~GB}$ of RAM.

The following computational, optimality and implementation issues are in order:

- We report that we have verified the well-functioning of the heuristic technique explained in Section III-B. To this end, the expected cost for dc-UC problem is obtained using the proposed Benders' algorithm. Its difference with respect to that of Case A (the non-decomposed solution) is verified to be smaller than $0.2 \%$.

- The computational times required for solving different instances are provided in the last column of Table VI. The computational time to solve Case B is comparatively higher. Note that both Cases B and C are solved iteratively, however the constraints of Case B are tighter. On the other hand, Case $\mathrm{A}$ is solved in a non-iterative way, thus its computational time is comparatively smaller.

- To speed-up convergence, suitable values for constants $\alpha^{\text {down }}, h^{\mathrm{Q}}$, and $h^{\mathrm{v}}$ need to be derived. In this paper, the values considered are $-10^{8}, 10^{3}$, and $10^{3}$, respectively, obtained through a trial-and-error analysis. Note that too large values of constants $h^{\mathrm{Q}}$ and $h^{\mathrm{v}}$ may lead to convergence fluctuations, while comparatively small values may lead to infeasible solutions.

- Note that the original ac-UC problem (1)-(3) is non-convex, and since the number of scenarios cannot be increased beyond a limit to ensure computational tractability, the result obtained may not be a global optimal. To overcome such issue, a multi-start decomposition approach is used [28]. Accordingly, the proposed decomposition problem is solved several times considering diverse initial points, and finally the one leading to the smallest value for the objective function (1) is selected. Among the diverse initial solutions checked, the flat start, i.e., $\mathbf{v}=1$ and $\boldsymbol{\theta}=0$, shows the best performance.

- To assess the effect of the considered tolerance $\varepsilon$ on the solution obtained, the proposed decomposition algorithm has been re-run considering a smaller value for $\varepsilon$, i.e., $0.1 \%$. The total cost of operation has not significantly changed: $\$ 651304.6$ for $\varepsilon=0.1 \%$ and $\$ 651909.9$ for $\varepsilon=0.3 \%$ (Case B). However, the number of iterations required for the convergence of Benders' algorithm and therefore the CPU time increases significantly with $\varepsilon=0.1 \%$.

- Note that reducing the number of scenarios is computationally appropriate if decomposition techniques are not used, while a larger number of scenarios is generally better if decomposition techniques are used. It is important to note that using a larger number of scenarios generally results in higher accuracy.

- Note that in the decomposed cases considered (Cases B and $\mathrm{C})$, the number of subproblems is $960(40 \times 24)$, being each of them comparatively small. The CPU time needed to solve each subproblem is about to $2 \%$ of the total time, which is reasonable. This means that the computational time may not significantly increase if a comparatively larger system is considered provided that the number of scenarios is reduced. However, to tackle case studies pertaining to real-world systems with thousands of nodes and lines and a large number of scenarios, the following additional alternatives are also available:

- To use a supercomputer,

- To implement parallelization techniques [29],

- To apply appropriate techniques to simplify the network [30] and/or to reduce the number of scenarios [31],

- To decompose the UC problem by area [32].

\section{CONClusions}

This paper proposes an efficient solution approach based on Benders' decomposition to solve a network-constrained ac-UC problem under uncertainty. The proposed approach allows decomposing such problem, which is mixed-integer nonlinear and generally intractable, into a mixed-integer linear master problem and a set of nonlinear but continuous subproblems, one per scenario and time period. Note that there is no off-the-shelf solver available for a MINLP problem, while MILP and NLP problems are both solvable using available commercial solvers. The numerical results obtained validate the well-functioning of the proposed approach.

We point out that the commitment status and dispatch results of generating units obtained by the dc and ac formulations might be different. The reasons for the differences are 1) the constraints that are not modeled in the dc-UC formulation, i.e., reactive power and voltage magnitude constraints, and 2) power losses which are not considered in the dc-UC formulation. It is also pointed out that the tightness level of voltage magnitude constraint in the ac-UC problem may potentially alter the commitment results. 


\section{APPENDIX A}

This appendix provides the functions used in this paper. Function $P_{n m t}^{\mathrm{DA}}(\boldsymbol{\theta})$ used in (2a) and (2h) is mathematically formulated as follows:

$$
P_{n m t}^{\mathrm{DA}}(\boldsymbol{\theta})=\frac{1}{x_{n m}}\left(\theta_{n t}^{\mathrm{DA}}-\theta_{m t}^{\mathrm{DA}}\right) \quad \forall n, \forall m \in \Omega_{n}, \forall t .
$$

Functions $P_{n m t s}(\mathbf{v}, \boldsymbol{\theta}), Q_{n m t s}(\mathbf{v}, \boldsymbol{\theta})$, and $S_{n m t s}(\mathbf{v}, \boldsymbol{\theta})$ used in (3a), (3b), and (3m) are mathematically formulated as follows:

$$
\begin{gathered}
P_{n m t s}(\mathbf{v}, \boldsymbol{\theta})=Y_{n m}\left[v_{n t s}^{2} \cos \left(\phi_{n m}\right)-v_{n t s} v_{m t s}\right. \\
\left.\cos \left(\theta_{n t s}-\theta_{m t s}-\phi_{n m}\right)\right] \quad \forall n, \forall m \in \Omega_{n}, \forall t, \forall s \\
Q_{n m t s}(\mathbf{v}, \boldsymbol{\theta})=-Y_{n m}\left[v_{n t s}^{2} \sin \left(\phi_{n m}\right)+v_{n t s} v_{m t s}\right. \\
\left.\sin \left(\theta_{n t s}-\theta_{m t s}-\phi_{n m}\right)\right] \quad \forall n, \forall m \in \Omega_{n}, \forall t, \forall s \\
S_{n m t s}(\mathbf{v}, \boldsymbol{\theta})=\sqrt{P_{n m t s}^{2}(\mathbf{v}, \boldsymbol{\theta})+Q_{n m t s}^{2}(\mathbf{v}, \boldsymbol{\theta})} \\
\forall n, \forall m \in \Omega_{n}, \forall t, \forall s .
\end{gathered}
$$

\section{APPENDIX B}

The dc version of ac constraints (3) included in the non-decomposed dc-UC problem (Case A of Section IV-B) is provided as follows:

$$
\begin{aligned}
& \sum_{i \in \mathcal{G}_{n}}\left(P_{i t}^{\mathrm{DA}}+r_{i t s}\right)+\sum_{k \in \mathcal{K}_{n}}\left(W_{k t s}-W_{k t s}^{\mathrm{SP}}\right) \\
& -\sum_{d \in \mathcal{D}_{n}}\left(L_{d t}^{\mathrm{P}}-L_{d t s}^{\mathrm{SH}}\right)=\sum_{m \in \Omega_{n}} \frac{1}{x_{n m}}\left(\theta_{n t s}-\theta_{m t s}\right) \forall n, \forall t, \forall s
\end{aligned}
$$

$$
\begin{aligned}
& (3 \mathrm{c})-(3 \mathrm{f}),(3 \mathrm{~h}),(3 \mathrm{i}),(3 \mathrm{k}),(3 \mathrm{l}) \\
& \frac{1}{x_{n m}}\left(\theta_{n t s}-\theta_{m t s}\right) \leq \bar{S}_{n m} \quad \forall n, \forall m \in \Omega_{n}, \forall t, \forall s .
\end{aligned}
$$

\section{ACKNOWLEDGMENT}

A. Nasri has been awarded an Erasmus Mundus PhD Fellowship. He would like to express his gratitude towards all partner institutions within the program as well as the European Commission for their support.

\section{REFERENCES}

[1] R. Baldick, "The generalized unit commitment problem," IEEE Trans. Power Syst., vol. 10, no. 1, pp. 465-475, Feb. 1995.

[2] F. Zhuang and F. D. Galiana, "Towards a more rigorous and practical unit commitment by Lagrangian relaxation," IEEE Trans. Power Syst., vol. 3, no. 2, pp. 763-773, May 1988.

[3] N. J. Redondo and A. J. Conejo, "Short-term hydro-thermal coordination by Lagrangian relaxation: solution of the dual problem," IEEE Trans. Power Syst., vol. 14, no. 1, pp. 89-95, Feb. 1999.

[4] A. J. Conejo, M. Carrión, and J. M. Morales, Decision Making Under Uncertainty in Electricity Markets, ser. Operations Research \& Management Science. New York, NY, USA: Springer, 2010.

[5] S. Takriti, J. R. Birge, and E. Long, "A stochastic model for the unit commitment problem," IEEE Trans. Power Syst., vol. 11, no. 3, pp. 1497-1508, Aug. 1996.

[6] P. Carpentier, G. Gohen, J.-C. Culioli, and A. Renaud, "Stochastic optimization of unit commitment: a new decomposition framework," IEEE Trans. Power Syst., vol. 11, no. 2, pp. 1067-1073, May 1996.
[7] J. M. Morales, A. J. Conejo, and J. Perez-Ruiz, "Economic valuation of reserves in power systems with high penetration of wind power," IEEE Trans. Power Syst., vol. 24, no. 2, pp. 900-910, May 2009.

[8] F. Bouffard, F. D. Galiana, and A. J. Conejo, "Market-clearing with stochastic security-Part I: formulation," IEEE Trans. Power Syst., vol. 20, no. 4, pp. 1818-1826, Nov. 2005.

[9] F. Bouffard, F. D. Galiana, and A. J. Conejo, "Market-clearing with stochastic security-Part II: case studies," IEEE Trans. Power Syst., vol. 20 , no. 4, pp. 1827-1835, Nov. 2005.

[10] Q. Wang, J. Wang, and Y. Guan, "Price-based unit commitment with wind power utilization constraints," IEEE Trans. Power Syst., vol. 28, no. 3, pp. 2718-2726, Aug. 2013.

[11] A. Kalantari, J. F. Restrepo, and F. D. Galiana, "Security-constrained unit commitment with uncertain wind generation: the loadability set approach," IEEE Trans. Power Syst., vol. 28, no. 2, pp. 1787-1796, May 2013.

[12] A. Tuohy, P. Meibom, E. Denny, and M. O'Malley, "Unit commitment for systems with significant wind penetration," IEEE Trans. Power Syst., vol. 24, no. 2, pp. 592-601, May 2009.

[13] J. Wang, M. Shahidehpour, and Z. Li, "Security-constrained unit commitment with volatile wind power generation," IEEE Trans. Power Syst., vol. 23, no. 3, pp. 1319-1327, Aug. 2008.

[14] W. S. Sifuentes and A. Vargas, "Hydrothermal scheduling using Benders decomposition: accelerating techniques," IEEE Trans. Power Syst., vol. 22, no. 3, pp. 1351-1359, Aug. 2007.

[15] Y. Fu, M. Shahidehpour, and Z. Li, "Security-constrained unit commitment with AC constraints," IEEE Trans. Power Syst., vol. 20, no. 2, pp. 1001-1013, May 2005.

[16] C. E. Murillo-Sanchez, R. D. Zimmerman, C. L. Anderson, and R. J. Thomas, "Secure planning and operations of systems with stochastic sources, energy storage, and active demand," IEEE Trans. Smart Grid, vol. 4, pp. 2220-2229, Dec. 2013.

[17] Q. P. Zheng, J. Wang, and A. L. Liu, "Stochastic optimization for unit commitment-a review," IEEE Trans. Power Syst., to be published.

[18] J. Ostrowski, M. F. Anjos, and A. Vannelli, "Tight mixed integer linear programming formulations for the unit commitment problem," IEEE Trans. Power Syst., vol. 27, no. 1, pp. 39-46, Feb. 2012.

[19] S. Muller, M. Deicke, and R. W. D. Doncker, "Doubly fed induction generator systems for wind turbines," IEEE Ind. Appl. Mag., vol. 8, pp. 26-33, May 2002.

[20] A. J. Conejo, E. Castillo, R. Minguez, and R. Garcia-Bertrand, Decomposition Techniques in Mathematical Programming: Engineering and Science Applications. Heidelberg, Germany: Springer, 2006.

[21] D. P. Bertsekas and N. R. Sandell, "Estimates of the duality gap for large-scale separable nonconvex optimization problems," in Proc. 21 st IEEE Conf. Decision and Control, Dec. 1982, vol. 21, pp. 782-785.

[22] Energy Information Administration [Online]. Available: http://www. eia.gov/countries/data.cfm.

[23] T. N. Santos and A. L. Diniz, "Feasibility and optimality cuts for the multistage Benders decomposition approach: Application to the network constrained hydrothermal scheduling," in Proc. IEEE Power Energy Soc. General Meeting, 2009 (PES '09), Jul. 2009, pp. 1-8.

[24] Reliability System Task Force, "The IEEE reliability test system-1996. A report prepared by the reliability test system task force of the application of probability methods subcommittee," IEEE Trans. Power Syst., vol. 14, no. 3, pp. 1010-1020, Aug. 1999.

[25] CPLEX, GAMS. The Solver Manuals. GAME/CPLEX, 1996 [Online] Available: http://www.gams.com/

[26] A. S. Drud, GAMS/CONOPT. Bagsvaerd, Denmark: ARKI consulting and development, 1996 [Online]. Available: http://www.gams.com/

[27] A. Brooke, D. Kendrick, A. Meeraus, R. Raman, and R. E. Rosenthal, GAMS, a User's Guide. Washington, DC, USA, GAMS Development Corp., Dec. 1998 [Online]. Available: http://www.gams.com/

[28] R. Mínguez, F. Milano, R. Zárate-Miñano, and A. J. Conejo, "Optimal network placement of SVC devices," IEEE Trans. Power Syst., vol. 22, no. 4, pp. 1851-1860, Nov. 2007

[29] A. Papavasiliou and S. S. Oren, "Multiarea stochastic unit commitment for high wind penetration in a transmission constrained network," Oper. Res., vol. 61, pp. 578-592, May 2013.

[30] X. Cheng and T. J. Overbye, "PTDF-based power system equivalents," IEEE Trans. Power Syst., vol. 20, no. 4, pp. 1868-1876, Nov. 2005.

[31] J. M. Morales, S. Pineda, A. J. Conejo, and M. Carrión, "Scenario reduction for futures market trading in electricity markets," IEEE Trans. Power Syst., vol. 24, no. 2, pp. 878-888, May 2009.

[32] A. Ahmadi-Khatir, A. J. Conejo, and R. Cherkaoui, "Multi-area unit scheduling and reserve allocation under wind power uncertainty," IEEE Trans. Power Syst., vol. 29, no. 4, pp. 1701-1710, Jul. 2014 
Amin Nasri (S'11) received the B.Sc. degree from Ferdowsi University, Mashhad, Iran, in 2006, the M.Sc. degree from Sharif University of Technology, Tehran, Iran, in 2008, and the (Ph.D.) Erasmus Mundus Joint Doctorate in Sustainable Energy Technologies and Strategies (SETS) from KTH Royal Institute of Technology, Sweden; Delft University of Technology, The Netherlands; and Comillas Pontifical University, Spain, in 2014.

$\mathrm{He}$ is currently a power system analyst at Sweco Energuide AB, Stockholm, Sweden. His research interests include power system operation, dynamics, stability and control, and FACTS.

S. Jalal Kazempour (S'08-M'14) received the B.Sc. degree from the University of Tabriz, Tabriz, Iran, in 2006, the M.Sc. degree from Tarbiat Modares University, Tehran, Iran, in 2009, and the Ph.D. degree from the University of Castilla-La Mancha, Ciudad Real, Spain, in 2013, all in electrical engineering.

In 2014, he was a postdoctoral fellow at the Whiting School of Engineering, Johns Hopkins University, Baltimore, MD, USA. He is currently a postdoctoral fellow at the Department of Electrical Engineering, Technical University of Denmark, Kgs. Lyngby, Denmark. His research interests include power systems, electricity markets, optimization, and its applications to energy systems.
Antonio J. Conejo (F'04) received the M.S. degree from the Massachusetts Institute of Technology, Cambridge, MA, USA, in 1987, and the Ph.D. degree from the Royal Institute of Technology, Stockholm, Sweden, in 1990.

$\mathrm{He}$ is currently a full Professor at The Ohio State University, Columbus, $\mathrm{OH}$ USA. His research interests include control, operations, planning, economics and regulation of electric energy systems, as well as statistics and optimization theory and its applications.

Mehrdad Ghandhari (SM'14) received the M.Sc. and Ph.D. degrees in electrical engineering from Royal Institute of Technology (KTH), Stockholm, Sweden, in 1995, and 2000, respectively.

$\mathrm{He}$ is currently a full Professor at KTH. His research interests include power system dynamics, stability and control, and FACTS and HVDC systems. 